\title{
Cervical Cancer Diagnosed During the Second Trimester of Pregnancy
}

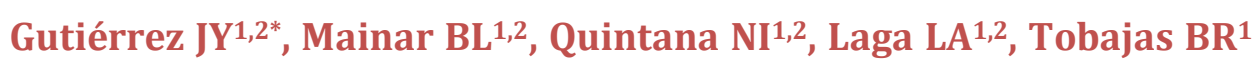 \\ and Ruiz Conde AM1,2 \\ ${ }^{1}$ Department of Gynecology, Miguel Servet University Hospital, College of Medicine \\ Zaragoza, Spain \\ ${ }^{2}$ IACS, Aragon Institute of Health Sciences, Zaragoza, Spain
}

\section{Case Report}

Volume 2 Issue 1

Received Date: March 29, 2017

Published Date: April 04, 2017

*Corresponding author: Yasmina José Gutiérrez, Department of Gynecology, Miguel Servet University Hospital, Number 1-3, Isabel la Catolica Road, Zaragoza, Spain, Tel: 657593282; Email: yjosegu@hotmail.com

\section{Abstract}

Cervical cancer during pregnancy is very uncommon. The mean age at diagnosis is 15 years less than the average age in general population. Approximately 3\% of cervical cancers are diagnosed during pregnancy, account for half of cancers diagnosed in this period. Management of a malignant process during pregnancy have special considerations to take into account like gestational age, disease stage and histology and desired preservation of the pregnancy. We present a case of diagnostic and treatment of microinvasive cervical cancer diagnosis in a woman at 14 weeks of gestation. After continuing with the pregnancy got a newborn baby healthy and disease-free a patient.

Keywords: Cervical Cancer; Pregnancy; Hysterectomy Laparoscopic

\section{Introduction}

Cervical cancer is the tumor with higher incidence and mortality in women after breast cancer around the world. $83 \%$ of cases occur in developing countries, and it is less common in countries with more resources. Spain is among the European countries with a lower incidence, but continues to be an unresolved issue since despite the campaigns of screening with a high fill, die every day in our country two women for this type of cancer. Cervical cancer is the most commonly diagnosed gynaecological malignancy during pregnancy. The incidence rates vary from 1-15 cases per 10.000 pregnancies [1].

Pregnancy is a good time to start or upgrade screen for cervical cancer in patients without routine gynaecologic checkups. In order to unify the guidelines on the prevention of cervical cancer in Spain, the Spanish Society of Gynecology and Obstetrics (SEGO) has developed a guide to clinical practice, published in 2014 [2].

Treatment in a pregnant patient with cervical cancer needs to be individualized an several issues are important like histological subtype, disease stag, nodal status, gestational age and obstetrical complications and desired preservation of the pregnancy, for this reason standardization of treatment is very difficult. In this situation of special vulnerability for women is important to make a proper advice based on current clinical guidelines, innovations and controversies [3].

We report a case of micro invasive cervical cancer in a woman at 14 weeks of gestation, and relate the diagnosis and treatment. 


\section{Case Report}

21-year-old patient referred to our service of Gynecology cancer prevention consultation, by the finding of atypical squamous cells of undetermined significance (ASC-US) and positive high-risk HPV. At the time of their referral to consultation was 14 weeks pregnant.

The anamnesis did not reveal the presence of any antecedent or surgical physician interest. She had a previous pregnancy with a vaginal delivery. Prior to the current PAP had never been performed. She was prostitute as a risk factor for cervical pathology.

A gynecologic examination at that time revealed in the ectocervix an exophytic polyp of $10 \mathrm{~mm}$ with suspicious appearance, dependent on cervical anterior lip, and excisional biopsy was performed for histopathological study. Colposcopy prior to removal of the polypoid formation showed ectocervix of normal appearance, with no major changes, only the polyp and its implementation base showed suspicious of malignancy. In the rectal examination not evidenced the existence of parametrial infiltration.

We performed the following tests:
- Transvaginal Ultrasound: cervix with normal size, undetected presence of other tumors to that level.

- Abdomino-pelvic Ultrasound: without pathological findings. Confirmed fetal vitality, with according to 14 weeks gestation fetus.

- Complete blood count, biochemistry and coagulation study: normal. Syphilis, Hepatitis B, Hepatitis C and HIV serologic test results are negative.

Histological examination to the excised cervical polypoid revealed papillary Squamous Cell Carcinoma with condilomatoso features. Study IHQ: Ck 7: positive. CK 20: negative. P16: positive at $2 / 3$ of the squamous epithelium external. CEA positive at the $2 / 3$ external squamous epithelium. Ki67: positive in $30 \%$ of the tumor cells. p53: weak over expression in $50 \%$ of the tumor cells. Not possible to assess correctly the infiltration by not displaying the base of the lesion, but the characteristics of the same they correspond to a particular type of tumor microinfiltrante-infiltrating. Figure 1 shows fragments of wall cervical Carcinoma squamous cell grows to form papillae and Figure 2 the immunohistochemical study of the tumor.

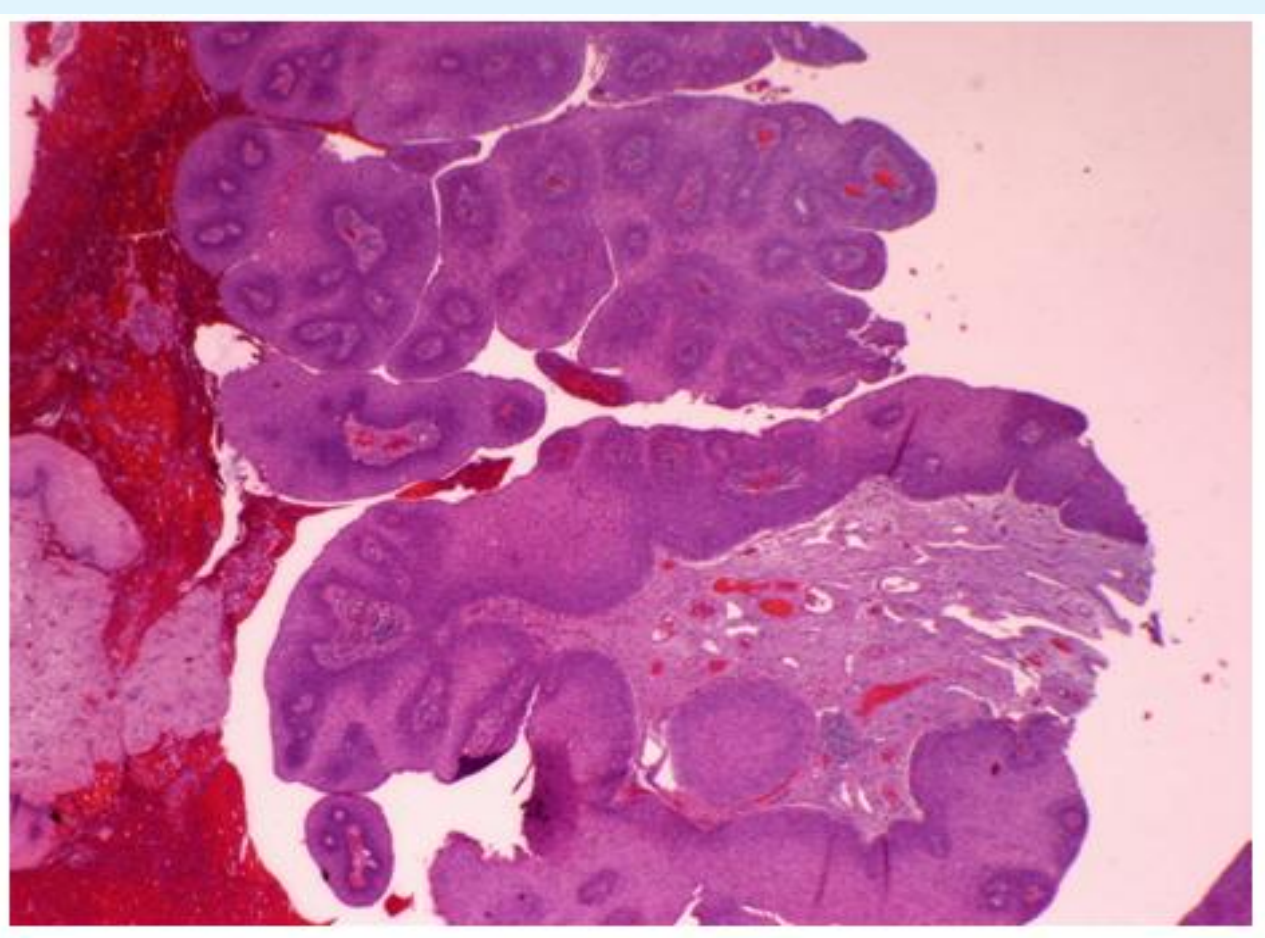

Figure 1: Fragments of wall cervical Carcinoma squamous cell grows to form papillae around an axis fibroconective and vascular. 


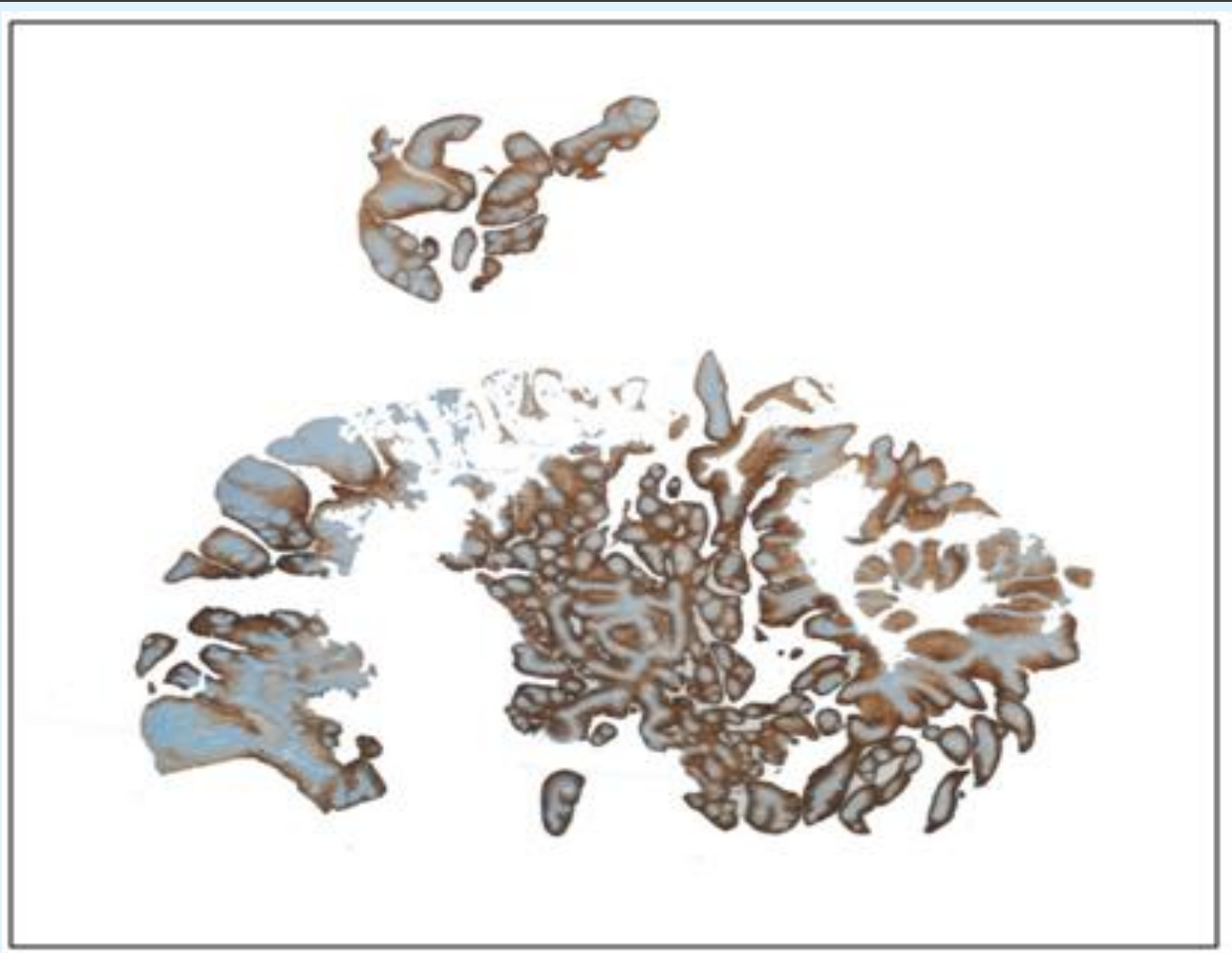

Figure 2: Immunohistochemical study of the tumor.

After informing the patient of the histopathological result and suggest a diagnostic excisional procedure such a conization to complete the study because the patient explicitly expressed the wish to preserve the pregnancy but she did not accept any kind of action, apart from a follow-up inquiry, during gestation. He made the decision despite explain the risks that could arise from the same. It only allowed complete the study and early treatment after birth

Trimestral disease stage evaluation showed in the gynecologic examination after polypectomy a cervix macroscopically normal, existing in the colposcopy only suspicious area very slight in the former base of the polyp implantation. Pregnancy passed without incident and without bleeding genitals of cervical origin.

In the gynecologic examination performed in the 38 week appeared new polypoid lesion in same place the removed previously of $6 \mathrm{~mm}$, excisional biopsy was performed with result of carcinoma papillary squamous areas condilomatosas in this biopsy warned very well the stroma, without observing signs of infiltracionmicroinfiltracion in the back part. At 39 weeks of gestation, a vaginal delivery of a male newborn, 3,430 g, with Apgar index of 9/10, was performed without any incidents.

The patient was examinated at 6 weeks postpartum, cytology was compatible with in situ carcinoma and confirmed by ectocervical biopsy. Cervical conization results: Carcinoma in situ with surface and glandular involvement with resection ends free, without evidence that currently signs of microinvasion. After informing the patient of these results and the different options to take, decided to perform a hysterectomy due to the resulting initial histopathological and having their reproductive wishes fulfilled.

We planned a simple total hysterectomy associated with laparoscopic pelvic lymphadenectomy and because of his age it was decided to retain both annexes. Figure 3 shows laparoscopic hysterectomy and we perform the technique without complications. 


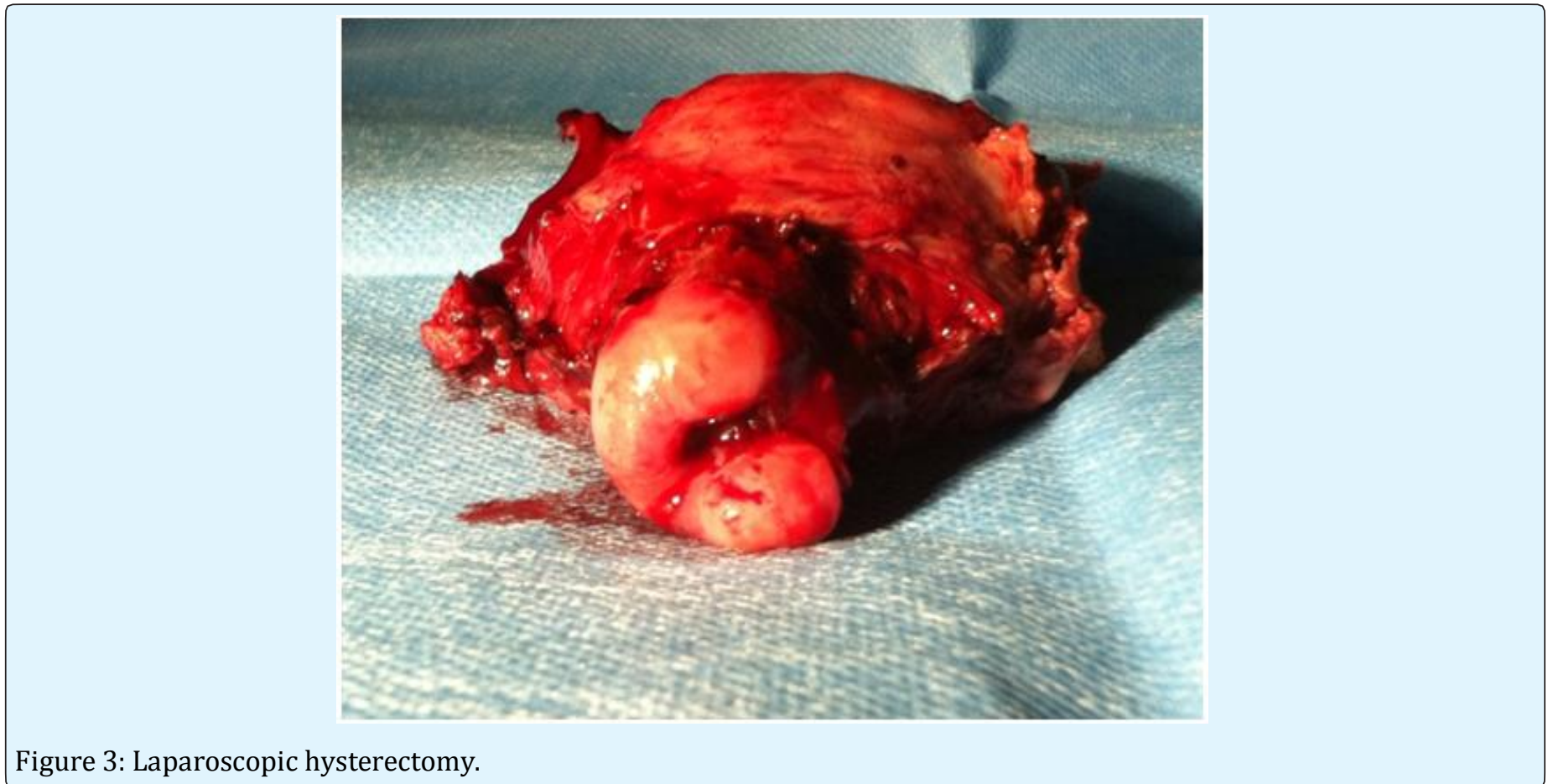

The result of the surgical specimen was: simple total hysterectomy without evidence of residual squamous papillary carcinoma. Injuries secondary to previous surgery: fibrosis, hemorrhage, extensive acute and chronic inflammation. Proliferative endometrium, Right pelvic lymphadenectomy with 10 lymph nodes without neoplastic signs and pelvic left with 9 nodes without affectation.

Gynecological cytology of the vaginal dome review was performed at 6 months: negative for intraepithelial lesion or malignancy. The patient is currently disease free 3 years of surgery.

\section{Discussion}

Cervical cancer ranks second in incidence and mortality after breast cancer in women worldwide, according to data from the World Health Organization (WHO). This cancer is typical of the middle ages of life as one third of cancers of the cervix occur in the reproductive period and up to $47 \%$ of invasive cancer diagnoses are made before the 35 years [4]. While the incidence of invasive cervical cancer is declining in most of the European countries thanks to the programs of screening, vaccination against the papilloma virus and changes in lifestyles in the population, continues to be a health major problem [5].
Cervical cancer during pregnancy is very uncommon. Pregnancy can mask cancer related symptoms with delay diagnostic and higher stages when cancer is diagnosed [6]. Report the reason for more frequent consultation is the genital bleeding, present in more than $50 \%$ of the cases, in the rest of the patients the diagnosis is usually done after the study by an altered cytology, as in our patient. However the majority of cervical cancers diagnosed during pregnancy are in the early stages as in our patient, $(76 \%$ in stage I cases), due to cervical exploration and cytology to form part of the routine prenatal revisions in many countries.

Karam et al. say pregnancy is an opportunity to screen for cervical cancer in patients without routine gynaecologic checkups [7-9]. Currently in Spain there is not a common policy of screening for cervical cancer, but that there are different public health strategies in all the autonomous communities. The majority of screening programmes are opportunistic, with non-optimal coverage and deficits in the equity and efficiency. It is estimated that more than $60 \%$ of the cervix tumours diagnosed relapse in women without prior screening or inappropriate screening. Establish a policy of population screening for cervical cancer, should be a priority according to the latest recommendations of the SEGO published in 2014 [2]. Some of the most significant changes included in the document refer to the change of 
Protocol of screening, by modifying the age of beginning at 25 years old.

We consider adequate the age at onset of screening, beginning at age 25 , as the incidence below this age is very low in our country and most of the cases it was rare histological as adenocarcinomas or sarcomas, for which cytology demonstrated a lower sensitivity. Our case is an exceptional case in a 21-year-old patient, probably both own pregnancy immunosuppression and the high number of sexual partners acted as risk factor for the development of the tumour.

The diagnosis of this disease during pregnancy always raises a number of ethical and therapeutic considerations that gestational age at diagnosis, stage, tumour size and genes desire are fundamental. Advice and treatment must be done with a multidisciplinary team and individually depending on the circumstances of each case [10-12].

There is evidence of retardation in the treatment of squamous cell carcinoma of the cervix in early stage is not likely to have a negative effect on the mother and that it could be extended to achieve fetal maturity, however the histologic subtypes of adenocarcinoma clear cell, small cell carcinoma or adenosquamous carcinoma have a faster growth than make recommended not to delay the start of the treatment $[13,14]$. In this case the patient decided to delay the treatment without negative consequences for your condition or for pregnancy, possibly because it is a squamous cell carcinoma.

Deliveries via vaginal in stage IB or more by having observed worst maternal outcomes such as hemorrhages are not recommended in relation to childbirth in these patients and implant in the place of the episiotomy [13]. In this case when it comes from an early stage were allowed a vaginal delivery that passed without incident. After the birth, it was decided to complete surgical treatment, not including the option of performing a transposition ovarian for not be foreseen the need for further treatment with radiotherapy.

The rationale for removal of the parametrium is to obtain a clear margin of the cervical tumour and to prevent local recurrence due to remaining cancerous parametrical lymph nodes. Several studies [15] have shown a parametrical involvement rate of $<1 \%$ in patients with tumour size $\leq 2 \mathrm{~cm}$, negative pelvic lymph nodes, and stromal invasion $\leq 10 \mathrm{~mm}$; this supports the option of less radical surgery without in patients that fulfill these criteria, for this reason we decided to perform in this case only a hysterectomy without parametrectomy.

\section{Acknowledgements}

This work was supported by the IACS, the Aragon Institute of Health Sciences and Source of FEDER cofinancing. Thank you for your support for our research group, Gynecology Oncology of the Miguel Servet Hospital.

\section{References}

1. Al-Halal H, Kezouh A, Abenhaim H (2013) Incidence and obstetrical outcomes of cervical intraepithelial neoplasia and cervical cancer in pregnancy: A population-based study on 8.8 million births. Arch Gynecol Obstet 284(2): 245-250.

2. Oncoguía SEGO: Prevención del Cáncer de Cuello Uterino (2014) Guías de práctica clínica en cáncer ginecológico y mamario. Publicaciones SEGO, Oncoguías.

3. Oncoguía SEGO: Cáncer de Cuello Uterino (2013) Guías de práctica clínica en cáncer ginecológico y mamario. Publicaciones SEGO, Oncoguías, pp: 1-28.

4. Ferlay J, Shin HR, Bray F, Forman D, Mathers C, et al. (2010) Estimates of worldwide burden of cancer in 2008: GLOBOCAN 2008. Int J Cance 127: 2893-2917.

5. Castellsagué X, Remy V, Puig- Tintoré LM, Sainz de la Cuesta R, González Rojas N, et al. (2009) Epidemiology and costs of screening and management of precancerous lesions of the cervix in Spain. J Low Genit Tract Dis 13(1): 38-45

6. Han SN, Mhallem Gziri M, Van Calsteren K, Amant F (2013) Cervical cancer in pregnant women: treat, wait or interrupt? Assessment of current clinical guidelines, innovations and controversies. Ther Adv Med Oncol 5(4): 211-219.

7. Karam A, Holschneider CH (2010) Cervical cancer in pregnancy. In: Basow DS \& Waltham, MA (Eds.). UpToDate.

8. Sellerest L, Mathevet P (2008) Precancerous cervical lesions during pregnancy: diagnostic and treatment. J Gynecol Obstet Biol Reprod 37(S1): S131-S138.

9. Cortés J, Martinón-Torres F, Cajal JMR, Gil A, Velasco J, et al. (2010) Prevención primaria y secundaria de los cánceres de cuello de útero y vulva: recomendaciones para la práctica clínica. Prog Obstet Ginecol 53(S1): 119. 
10. Cancer of the cervix and pregnancy. Assistance protocols in Obstetrics. Revised Spanish Recommendations 2008. Spanish Society of Gynecology and Obstetrics (SEGO).

11. Petterson BF, Andersson S, Hellman K, Hëllstrom AC (2010) Invasive carcinoma of the uterine cervix associated with pregnancy: 90 years of experience. Cancer 116(10): 2343-2349.

12. Favero G, Chiantera V, Oleszcuk A, Gallota V, Hertel H, et al. (2010) Invasive cervical cáncer during pregnancy: laparoscopic nodal evaluation before oncologic tratment delay. Gynecol Oncol 118(2): 123127.
13. Lee JM, Lee KB, Kim YT, Ryu HS, Kim YT, et al. (2008) Cervical cancer associated with pregnancy: results of multicenter retrospective Korean study (KGOG1006). Am J Obstet Gynecol 198(1): 92e1-e6.

14. Adriaensen WJ, Mathei C, Buntinx FJ, Arbyn M (2013) A framework provided an outline toward the proper evaluation of potential screening strategies. J Clin Epidemiol 66(6): 639-647.

15. Halaska MJ, Rob L, Robova H, Cerny M (2016) Treatment of gynecological cancers diagnosed during pregnancy. Future Oncol 12(19): 2265-2275. 